\title{
A Wideband Planar Antenna for In-body Imaging
}

\author{
R. Nilavalan ${ }^{(1)}$, I. J. Craddock*(1) J. Leendertz ${ }^{(1)}$, A. Preece ${ }^{(1)}$, R. Benjamin ${ }^{(2)}$ \\ (1) University of Bristol, UK \\ (2) 13 Bellhouse Walk, Bristol, UK
}

\section{Introduction}

Breast cancer is the most common cancer in women. $\mathrm{X}$-ray mammography is currently the most effective detection technique, however it suffers from a relatively high missed- and false-detection rates, involves uncomfortable compression of the breast and also entails exposure to ionizing radiation.

Microwave detection of breast tumours is a potential non-ionising alternative being investigated by a number of groups [1][2]. In these microwave-based systems, in a similar fashion to Ground Penetrating Radars, microwaves are transmitted from an antenna or antenna array, and the received signals, which contain reflections from tumours, are recorded and analysed.

A pre-requisite for all of these systems is a suitable antenna (e.g. [3]). This contribution presents in detail for the first time, an inexpensive, wideband, planar antenna design, specifically designed to radiate into breast tissue.

\section{Design Process}

The chosen frequency band was in the range 5 to $10 \mathrm{GHz}$, this bandwidth giving the potential of good resolution in tissue, when combined with a large array aperture. A bandwidth of $67 \%$ was therefore the design target.

A printed antenna design was seen as desirable, for reasons of costs, complexity and the ability to manufacture devices to the close tolerances required. Stacked patch antennas are well-known to have good operating bandwidths, although the bandwidths achieved are usually only of the order of $20 \%$ [4]. A target of $67 \%$ was therefore quite ambitious.

Operating bandwidth for stacked patches is often limited by the fact that the lower patch is usually probe-fed, and for this reason a slot-fed approach was chosen. Figure 1 shows the configuration employed, with a microstrip line used to feed the patch through a slot in the antenna ground plane.

Most stacked patches are of course designed for operation in air, using low dielectric materials (often including a foam or air centre layer), however here it is necessary to radiate into a high dielectric medium (the breast - with a typical 
relative permittivity of 10 ). The decision was therefore taken to build the antenna on higher-dielectric materials.

Dimensions were optimised using an in-house FDTD code in order to obtain the best matching. The final design consists of two patches (designed to resonate at either end of the frequency range and therefore having different widths of $6 \mathrm{~mm}$ and $9 \mathrm{~mm}$ ) printed on either sides of a $0.8 \mathrm{~mm}$-thick dielectric substrate of $\varepsilon_{\mathrm{r}}=2.2$. This substrate is separated from the ground plane by a $1.9 \mathrm{~mm}$-thick second substrate of $\varepsilon_{\mathrm{r}}=10.2$. A final thin layer of $\varepsilon_{\mathrm{r}}=10.2$ (not shown, for clarity, in Figure 1) is often in practice employed as a radome on the top of the structure.

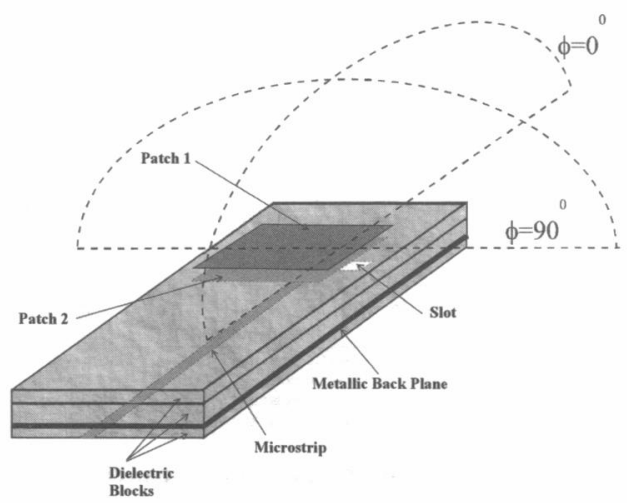

Figure 1: Antenna structure

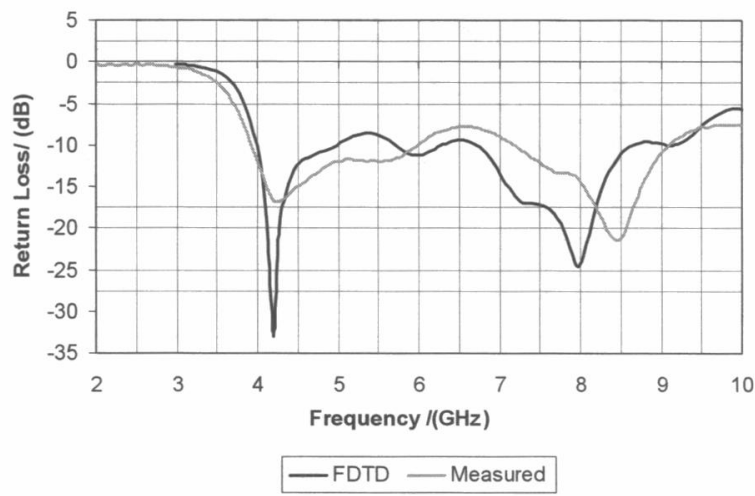

Figure 2: Antenna input response 
This is close to the desired band of operation and the antenna was constructed using printed circuit techniques and measured experimentally while radiating into a breast tissue phantom. Agreement between FDTD and experimental results in Figure 2 is very pleasing, especially given the inevitable manufacturing tolerances involved in defining the patches and feed, and then undertaking the blindalignment of the different substrate layers.

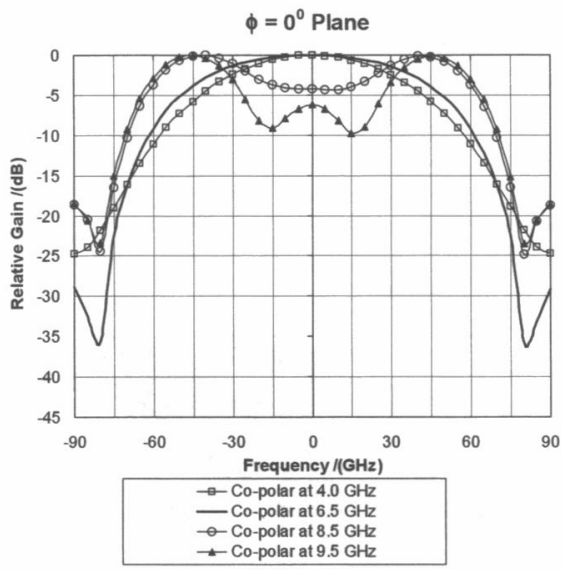

Figure 3: Calculated radiation pattern in the medium for $p h i=0$ plane

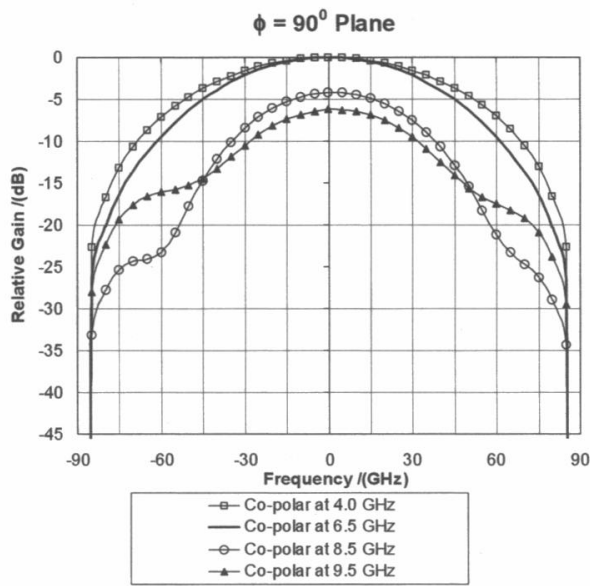

Figure 4: Calculated radiation pattern in the medium for $\mathrm{phi}=90^{\circ}$ plane 
Practical patterns measurement is difficult, since the antenna must be measured in medium, rather than air, therefore FDTD simulations were again used. The farfield radiation patterns of the stacked patch antenna were found by post processing the FDTD frequency domain data at specific frequencies. The calculated co-polar radiation patterns for the principal planes are shown in Figure 3 and Figure 4. Cross-polar levels (not shown) were 40dB lower.

The patterns can be seen to be relatively stable over frequency with the exception of the $9.5 \mathrm{GHz}$ result in Figure 3, where a null begins to form in the pattern. Front-to-back ratios were also calculated by integrating the power in the pattern over the front and back half-spaces - typically a $15 \mathrm{~dB}$ ratio is obtained, as seen in Table 1.

\begin{tabular}{|c|c|c|c|c|c|c|}
\hline Frequency/ (GHz) & 4.0 & 5.5 & 6.5 & 8.0 & 9.0 & 9.5 \\
\hline $\begin{array}{c}\text { Power Ratio- } \\
\text { Far fields/ (dB) }\end{array}$ & 14.4 & 18.1 & 15.2 & 14.5 & 11.5 & 11.5 \\
\hline
\end{tabular}

Table 1: Antenna front-to-back power ratio

\section{Conclusions}

The stacked-patch antenna presented herein has an excellent input bandwidth of approximately $77 \%$ and a beamwidth of approximately $\pm 45^{\circ}$ in $\phi=0^{\circ}$ plane and $\pm 35^{\circ}$ in $\phi=90^{\circ}$ plane at $6.5 \mathrm{GHz}$, calculated in $\varepsilon_{\mathrm{T}}=9.8$ medium. Although there is a slight degradation in the pattern at the upper end of the frequency range, the achieved bandwidth was in excess of expectations and, in being inexpensive to construct and readily integrated into an array, the design is well-suited to the intended application [5].

\section{References:}

[1] X. Li, S. Hagness, B. D. Van Deen, D. van den Weide, "Experimental investigation of Microwave Imaging via Space-Time Beamforming for Breast Cancer Detection," Proc. IEEE International Microwave Symposium, vol. 1, pp 379-382, 2003.

[2] E. C. Fear, J. M. Sill, "Preliminary Investigations of Tissue Sensing Adaptive Radar for Breast Tumor Detection", Proceedings of the 25th Annual International Conference of the IEEE Engineering in Medicine and Biology Society, pp 37873790, 2003.

[3] S. C. Hagness, A. Taflove, and J. E. Bridges, "Three-dimensional FDTD analysis of a pulsed microwave confocal system for breast cancer detection: Design of an antenna-array element," IEEE Trans. Antennas and Propagation, vol. 47, pp. 783791, May 1999

[4] I. J. Craddock, D. L. Paul, C. J. Railton, G. Ball \& J. Watts, "A CylindricalCartesian FDTD Model of a 17-Element Conformal Antenna Array", Electronics Letts., vol. 37, pp. 1429-1431, Nov. 2001.

[5] I. J. Craddock, R. Nilavalan, J. Leendertz, A. Preece, R. Benjamin, "Experimental Investigation of Real Aperture Synthetically Organised Radar for Breast Cancer Detection," submitted to the IEEE AP-S International Symposium and USNC/URSI National Radio Science Meeting, Washington DC, July 2005. 